\title{
Abordaje docente y administrativo de los conflictos estudiantiles en el campo de la educación secundaria
}

\author{
High School conflicts its approach by the administration and teaching \\ staff
}

\author{
Lisbeth Chacón Soto \\ chacon,lisbeth@gmail.com \\ Yirlany Elizondo Vásquez \\ yir.yila@gmail.com \\ María Isabel Fernández Ramírez \\ isafer_ra@hotmail.com \\ Jesusita Triana Mora \\ trianamora@yahoo.es \\ Eva María Umaña Vásquez \\ emuva@hotmail.com \\ Ministerio de Educación Pública, Costa Rica
}

Recibido: 17 setiembre 2010 Aceptado: 01 noviembre 2010 Corregido: 29 junio 2011

\section{Resumen}

Este artículo presenta el estudio sobre el "Abordaje docente y administrativo de los conflictos estudiantiles en el campo de la educación secundaria”. En este se ofrecen datos sobre situaciones actuales a nivel mundial y nacional de conflictos en el ámbito social y educativo. La investigación se apoya en documentos bibliográficos que brindan una visión integral acerca del reto de quienes administran las organizaciones educativas, ante el abordaje oportuno de los conflictos estudiantiles, para el cumplimiento de los fines y propósitos de la educación costarricense. Así mismo, se plantean los resultados extraídos de este estudio y se sugieren estrategias para el abordaje preventivo.

Palabras Claves: administración de la educación/ conflictos estudiantiles/ violencia en centros educativos /calidad de la educación/ sociología de la educación/ adolescencia.

\footnotetext{
Abstract

This paper presents a study on "Administrative approach to teaching and student conflict in the field of secondary education". Data on current global situation and national conflicts in the social and educational is presented first.

The research is based on bibliographic documents which provide a comprehensive view about the challenge of those who administer education, with emphasis in conflicts
} 
that students experience when trying to fulfill the expectations and purposes of education in Costa Rica. It also contains the results drawn from this study and suggests strategies for a preventive approach.

Keywords: educational administration, student conflict, violence in schools, quality of education, sociology of education, adolescence.

\section{Introducción}

Dada la complejidad de la actual sociedad, son muchos los desafíos que requieren respuestas inmediatas y acertadas. En este campo, al sistema educativo se le delegan grandes responsabilidades como agente formador de la sociedad, ante lo cual debe responder desde un adecuado modelo de administración de la educación.

Bajo la influencia del actual momento histórico, social, político y económico de tendencia globalizante, en un mundo que cada vez se hace más cercano caracterizado por la mundialización de la economía, lo que ha traído consigo el incremento de la pobreza, individualismo, desempleo, conflictos armados, xenofobia y otros.

Las poblaciones juveniles y de infantes son quienes conforman un gran porcentaje de la ciudadanía civil, consideradas poblaciones vulnerables. Las organizaciones educativas evidencian esta realidad, por lo que, es frecuente, últimamente, escuchar información relacionada con diferentes problemas, dentro de los cuales se hace referencia a los conflictos en los centros escolares, generalmente descargan en actos de violencia o agresiones, que quizás pudieron haberse evitado, y que generan un impacto negativo en el proceso educativo que se ejecuta en las organizaciones educativas. Ante este entorno, el sistema educativo costarricense no está exento de los conflictos estudiantiles en sus centros educativos:

El incidente en el que dos escolares recibieron heridas de armas de fuego mientras se hallaban bajo la “protección” de una escuela, provocó naturales reacciones de asombro e indignación. (Durán, 2004, p. 34) 
Toda esta situación se ve reflejada en las diferentes conductas de los jóvenes, generalizando conflictos que, de no ser abordados adecuadamente, podrían desencadenar en violencia.

En la actualidad, la administración de la educación se presenta como un área de conocimiento humano con complejidades y desafíos.

Las organizaciones son diferentes y es por ello que quien las administra debe tener las capacidades y habilidades necesarias para desempeñarse efectivamente en los niveles de la administración.

Esta efectividad es valorada, principalmente, por lo que el personal directivo logre, transformando todo su conocimiento en acción de cambio.

El debate emergente sobre los conflictos estudiantiles reafirman la importancia de analizar este tema en las instituciones educativas. Este campo de análisis ha tomado últimamente mayor auge en la investigación educativa debido a su incremento, considerándose que debe ser materia de interés para quienes administran las organizaciones educativas. A escala mundial diversas instituciones se han preocupado por incentivar la paz y eliminar el conflicto entre los seres humanos, sobre todo en el contexto educativo, esto ha sido abordado por investigadores a nivel internacional.

A pesar de que diferentes disciplinas sociales y educativas han estudiado la temática, es importante enfocarlo hacia las instituciones educativas de secundaria, estos centros educativos representan a una población vulnerable debido a las características propias de la etapa de la adolescencia de su población estudiantil. De igual forma, es indispensable analizar el papel preponderante de la administración de la educación en el abordaje de los conflictos estudiantiles, principalmente debido a que el tema se ha dirigido poco hacia esta población.

Sobre esta problemática y contexto, surgió la necesidad de investigar el abordaje de los conflictos estudiantiles en las organizaciones educativas, en este caso en un centro educativo de educación secundaria público, de modalidad técnica, que pertenece al distrito de Jacó, del circuito 05 de la Dirección Regional de Educación de Aguirre, ubicada en el Cantón de Garabito, denominado Colegio Técnico Profesional de Jaco, ubicado en la provincia de Puntarenas, San José, Costa Rica. Este colegio es una organización que en la 
actualidad se enfrenta a variados problemas de conflictos estudiantiles, que en su mayoría podrían estar relacionados con una marcada multiculturalidad de influencia extranjera, que pareciera no ser siempre positiva y repercutir en la promoción de antivalores, y conductas inapropiadas aprendidas de diversas culturas. Así mismo, esta institución presenta una serie de problemas sociales, propios de su contexto social de ubicación, tales como prostitución y una comunidad inmersa en la drogadicción.

Es por lo anterior, que este estudio pretende dar respuesta a interrogantes relacionadas con el abordaje de los conflictos estudiantiles, desde la administración de la educación. Estas interrogantes encontrarán respuesta mediante los siguientes objetivos de la investigación:

\subsection{Objetivos}

\subsubsection{Objetivos Generales}

a. Analizar el abordaje de los conflictos estudiantiles por parte del personal administrativo, docente y técnico docente del Colegio Técnico Profesional de Jacó.

b. Plantear estrategias, para el abordaje efectivo de los conflictos estudiantiles en el Colegio Técnico Profesional de Jacó, que sirvan como herramientas de apoyo para la Administración de la Educación.

\subsubsection{Objetivos Específicos}

a.1 Identificar las manifestaciones de conflictos estudiantiles que se generan en la institución.

a.2 Determinar los factores internos que influyen en los conflictos estudiantiles.

a.3 Identificar las estrategias que utilizan los docentes, orientador y administrador educativo en la resolución de los conflictos estudiantiles.

a.4 Identificar los principales problemas sociales de la comunidad de Garabito.

b.1 Diseñar una estrategia que facilite la resolución alternativa de conflictos estudiantiles en el Colegio Técnico Profesional de Jacó. 


\section{3. Problemas sociales del contexto del centro educativo en estudio}

El diagnóstico Institucional de esta organización educativa del 2007, señala los principales problemas sociales que presenta el cantón de Garabito, en el que se ubica este centro educativo, entre los que se encuentran:

- Drogadicción: en esta comunidad es muy común observar cómo los niños y niñas, desde edades tempranas, tienen sus primeros acercamientos a varios tipos de drogas, esto provocado por diferentes razones, entre las que están:

a. Influencia de extranjeros, que encuentran en Jacó un paraíso donde consiguen todo tipo de droga y a muy bajo precio.

b. Narcotráfico: Jacó es uno de los pueblos de Costa Rica donde se consigue cualquier tipo de estupefaciente, que se venden en diferentes sitios y hasta en centros educativos o lugares circunvecinos.

c. Falta de información: los niños y niñas, jóvenes y personas adultas de esta comunidad presentan grandes deficiencias en el conocimiento de las repercusiones que genera la droga en el organismo de las personas y en la comunidad en sí.

- Prostitución: Jacó se ha convertido en un centro donde se lleva a cabo la prostitución, tanto de mujeres propias de la zona, como de extranjeras que encuentran en esta ciudad un lugar idóneo para laborar. En los últimos años este problema ha comenzado a tocar a la niñez, dado que personas inescrupulosas tratan de ganar dinero prostituyendo a menores.

- Pobreza: Es evidente la brecha entre las clases sociales, se observan personas muy solventes económicamente, pero a la vez existen barrios donde la pobreza impera, especialmente en aquellos donde habitan personas nicaragüenses.

\subsection{Referente metodológico}

Para el desarrollo de la investigación se seleccionó un diseño fenomenológico. Según Barrantes (2005), el diseño de la fenomenología pretende conocer los significados 
que resultan de las experiencias de los individuos y ver las cosas desde el punto de vista de otras personas, describiendo, comprendiendo e interpretando. Tomando en cuenta la participación de los principales actores de dicha institución educativa (estudiantes, docentes, orientadoras y director), por ser una investigación basada en la comprensión y en la visión de mundo de la población involucrada propio del principio de la fenomenología se utilizó un enfoque de investigación cualitativa.

\subsubsection{Población y Sujetos de estudio}

En el estudio participaron 18 docentes guías, 18 estudiantes presidentes del total de secciones de la institución, dos orientadoras y el director del centro educativo.

\subsubsection{Fuentes de información:}

Primarias: director o asistente de dirección, orientadoras, docentes guías y estudiantes.

Secundarias: se indagó en documentos que la institución posee como: "Reglamento de Evaluación de la Conducta” y “Reglamento Interno de Evaluación”

\subsubsection{Técnicas e Instrumentos de Recolección de la información}

Se requirió para la recolección de información de tres tipos de poblaciones (administración, personal docente y alumnado) que difieren en: edad, interés, objetivos, puesto dentro de la organización, capacidad para percibir situaciones, motivo por el que se elije un instrumento y una técnica para cada tipo de población. Dichos instrumentos y técnica fueron debidamente validados.

En el caso del administrador se eligió la entrevista en profundidad ya que el espacio físico (oficina) y la disponibilidad de tiempo por su parte favorecieron la aplicación de dicho instrumento.

Al personal docente y de orientación se optó utilizar el cuestionario ya que cada actor elije el momento y lugar conveniente para contestar. 
Por ser una población numerosa, de diferentes edades y por la necesidad de ser dirigidos, con el alumnado se utilizó la técnica de grupo focal.

\subsection{Referentes teóricos}

El presente apartado desarrolla aquellos fundamentos teóricos esenciales para abordar el tema de los conflictos estudiantiles desde la administración de la educación. Es así que se hace una revisión de la teoría de la educación, teoría de la administración, administración de la educación, sociología de la educación, calidad de la educación y conflictos estudiantiles. Con el propósito de obtener un mayor conocimiento sobre estos temas, se inicia con una contextualización de las demandas y retos actuales que enfrenta la educación.

\subsubsection{Teoría de la Educación}

La teoría científica es la base de una ciencia compuesta por leyes y reglas sistemáticamente organizadas para explicar los fenómenos que se interrelacionan con ella; sin embargo, la teoría de la educación se encuentra muy alejada de ser un sistema rígido, ya que, alude al marco del pensamiento que estructura y guía cualquier actividad teórica (Carr, 1996).

La educación es un término antiguo, conocido y utilizado en todo momento histórico y cultural, tal y como lo expresa Sarramona (citado por Cordero, 1990):

La educación es tan antigua como el hombre. En efecto, desde su aplicación el hombre se preocupó de “criar” y “cuidar” a sus hijos hasta que pudieran valerse por sí mismos. En esta función educativa inicial puede verse la raíz etimológica misma del concepto de educación. (p.43)

Se visualiza así que la educación es un proceso que se da en todo momento del desarrollo del ser humano, buscando su máximo progreso como persona. 
Argudín (2006) concuerda al afirmar que el ser humano vive una continua transformación durante el proceso de aprendizaje, cuando aporta su concepto de educación:

(...) la educación se entiende como el proceso donde se realiza la unión de dos actividades: la enseñanza y el aprendizaje. Se puede definir la enseñanza como la acción del educador sobre los educandos (programada o no), ésta tiende a transformar al alumno a partir de que este es capacitado. El aprendizaje es el resultado demostrable de la labor que realiza el alumno para adquirir instrucción. El ser humano tiene la capacidad de aprender y por eso puede ser educado. (p.26)

La cita anterior demuestra que aprendizaje y educación no son sinónimos. Para enriquecer el aporte mencionado anteriormente, Apple (1997) manifiesta que la educación es lo que queda después de haber olvidado lo que se aprendió. La adquisición de la educación es tan intensa como la disposición de aprender a posesionarse de las conductas y filosofías aptas del contexto, interiorizarlas y aplicarlas y, así mismo, la capacidad de desechar e ignorar lo inapropiado.

La admnistración de la educación lleva sobre sí la importante responsabilidad de capturar, bajo su óptica, el nuevo reto de atender estratégicamente los conflicos que emergen dentro del ambiente de las organizaciones educativas.

\subsubsection{Administración y administración de la educación}

La administración teóricamente implica:

Interpretar los objetivos propuestos por la organización y transformarlos en acción organizacional, a través de la planeación, organización, la dirección y el control de todos los esfuerzos realizados en las áreas y en todos los niveles de la organización, con el fin de alcanzar tales objetivos de la manera más adecuada a la situación y 


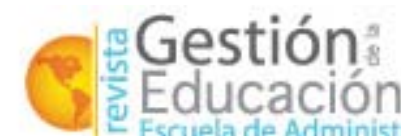

garantizar la competitividad en un mundo de negocios muy competitivo y complejo. (Chiavenato, 2006, p. 10)

Estos principios científicos, aunque corresponden a la teoría de la administración en general, son aplicables al campo de la educación, donde se hace necesario el logro de una educación de calidad mediante acciones de planificación y control principalmente. Por tal razón, es igualmente importante, en términos de calidad, dar un carácter científico a la administración de la educación.

En vista de que uno de los objetivos fundamentales de la administración de la educación es ofrecer los mejores servicios educativos, a través de una óptima utilización de los recursos que la sociedad pone a su disposición, mediante la acción planificada de los objetivos organizacionales, es que se vuelve indispensable el papel del profesional en administración de la educación, porque los recursos humanos y materiales requieren de un adecuado desempeño, con el fin de contribuir con el logro satisfactorio de los objetivos

La labor de la administración de la educación es compleja y variada, por lo que requiere de la función especializada de las personas que cumplen con esa tarea. Algunos teóricos se refieren a las características más importantes que describen el perfil del profesional en administración de la educación, las cuales se desarrollan seguidamente.

La administración de la educación tiene un papel directivo dentro de la institución que debe ser desempeñado con liderazgo, procurando la funcionalidad y calidad de los procesos que ahí se gestan, estos tienen su razón de ser en el aspecto pedagógico. Sin embargo, las funciones son diversas y las habilidades también deben serlo, para responder con éxito a las demandas y retos que impone la sociedad actual.

Todo profesional en administración de la educación para desempeñar efectivamente su función conductora dentro de la institución, debe tener un temperamento equilibrado, tolerancia a la crítica, seguridad en sí mismo y capacidad de decisión, una continua autoevaluación de su quehacer mediante una actitud reflexiva de actuación con las normas establecidas, facilidad de comunicación, liderazgo y agilidad en la conducción de grupos. 
Como complemento, a lo largo de la formación profesional y de la experiencia laboral en la administración de la educación, se deben desarrollar capacidades o habilidades para enfrentar con éxito las diversas situaciones que se le presentan. Al respecto, García, Rojas y Campos (2002) señalan algunas de ellas:

- Capacidad para captar las totalidades dinámicas, que le permita establecer objetivos y políticas para impulsar proyectos

- Capacidad para delegar mediante la transferencia planificada de autoridad y responsabilidad a otros, dando seguimiento y apoyo oportuno.

- Capacidad para armar equipos de trabajo eficaces a los cuales se debe ser capaz de asignar roles y tareas, niveles de autonomía e iniciativa, así como compromiso y responsabilidad.

- Capacidad para generar la circulación sistemática de la información, para contrarrestar el efecto negativo del rumor, que se presenta comúnmente en las organizaciones.

- Capacidad para profesionalizar el rol y enriquecerlo con aportes del campo organizativo, alejando la gestión del líder de la improvisación.

- Capacidad para conducir en coherencia con los valores democráticos, procurando la participación.

- Capacidad para trabajar con ética y compromiso ante las situaciones influidas por el contexto.

Aunado a estos aspectos de personalidad y de desarrollo de destrezas para la conducción del centro educativo, se presenta una habilidad que se debe tomar en cuenta para responder a los múltiples retos que involucran la administración efectiva de las organizaciones educativas, donde la presencia de los conflictos no es ajena a la dinámica escolar, ya que estos son parte de la vida de las personas, tanto a nivel intrapersonal como social. Así lo describen García, Rojas y Campos (2002), al mencionar que el conflicto en la escuela no debe evitarse ni ignorarse, sino enfrentarse para su adecuada resolución. Esta labor de resolución de los conflictos en el contexto educativo requiere también de ciertas 
habilidades, presentes en el perfil del profesional en administración de la educación, las autoras mencionan las siguientes:

- Acepta que el conflicto es parte de los grupos humanos

- Conoce sobre la naturaleza, tipologías y comportamientos de personas involucradas en los conflictos.

- Proporciona capacitación constante al personal, sobre estrategias e información, para el abordaje optimo de los conflictos..

- Aborda el conflicto, mediante la comunicación abierta, con el fin de obtener un resultado positivo.

- Orienta el abordaje de manera imparcial y separa a las personas del problema.

Una de las estrategias en la administración de la educación que se puede implementar para el abordaje efectivo de las situaciones conflictivas dentro de las organizaciones educativas, puede estar relacionada con la toma de decisiones; así, lo recomiendan García, Rojas y Campos (2002) al sugerir que se realice, inicialmente, un diagnóstico de la situación y posteriormente, una toma de conciencia acerca de la situación problemática existente y de los elementos involucrados. Debe darse también, como paso siguiente, el análisis del problema y la búsqueda de soluciones, para lo cual es apropiado el uso de diferentes técnicas que propicien la participación activa de las partes involucradas.

\subsubsection{Sociología de la educación}

Los problemas sociales se definen como toda fractura que existe en la sociedad y que obstaculiza el adecuado proceso formativo del individuo y por ende repercute negativamente en el proceso educativo. Delval (2002):

Los problemas educativos básicos no se circunscriben al ámbito de la escuela, sino que van mucho más allá. Son los problemas de la sociedad en general. Y como no se sabe, o no se quiere, resolver esos problemas de la sociedad en general se pretende que los resuelva la escuela, lo cual resulta a todas luces imposible. Se 
hace referencia al aumento de la delincuencia juvenil, la falta de valores morales, la adopción de conductas inadecuadas, y se atribuye a la escuela la posibilidad de cambiar eso. (p.62)

Los problemas sociales no pueden ser resueltos desde la organización educativa, es por eso que resulta una tarea compleja la administración de los centros educativos ante el aparente crecimiento de los mismos. Para realizar con éxito esta labor es importante apoyarse en disciplinas que ayudan a estudiar estos fenómenos sociales y darles respuestas.

En la sociología de la educación se plantea el problema de la relación entre educación y sociedad. Es decir, el papel de la educación como agente de influencia social, esta relación educación-sociedad define el ámbito científico de la sociología de la educación.

No es posible separar la educación del contexto social, esta vinculación repercute en las conductas del estudiantado y en las relaciones que se establecen dentro de las organizaciones educativas y de estas con la sociedad.

Es importante recalcar la influencia del contexto social con las organizaciones educativas.

El tema de la calidad de la educación no puede dejarse fuera de este debate teóricopráctico, es por ello que seguidamente se aborda, a nivel general.

\subsubsection{Calidad de la educación}

El tema de la calidad de la educación es tan complejo, como sus abordajes y momentos históricos en que se construye teóricamente.

La calidad de la educación no es responsabilidad directa de la organización educativa, sino que es regida por las disposiciones gubernamentales, así se establece en la Constitución Política y en todas las leyes y reglamentos colaterales al sistema educativo.

Para el caso del sistema educativo costarricense, y dadas las características sociales del Estado, la educación sigue siendo una responsabilidad social, según se plasma en la 
Constitución Política (1949), en sus artículos 77 y 78, al expresar que la educación será un proceso desde preescolar hasta la educación superior, la educación básica es gratuita y obligatoria y la preescolar y superior es gratuita y costeada por el estado.

Además de lo expresado en la Constitución Política (1949), el sistema educativo costarricense, mediante sus fines educativos, también enmarca los parámetros sobre los cuales la educación debe encaminarse.

Al contemplar la Ley Fundamental de Educación (1957), se observan las más nobles aspiraciones de calidad para la educación costarricense. Según el artículo uno, el Estado se asegura una ciudadanía alfabetizada en su totalidad y aspira a la formación integral del ciudadano a través de la educación. En el artículo dos, expresa en sus fines la formación de ciudadanos amantes de su patria que conozcan sus derechos y deberes y aptos para un desenvolvimiento y convivencia en una sociedad democrática. Un sistema escolar eficaz y de calidad es el que maximiza la capacidad de las escuelas para alcanzar los resultados.

Para Garbanzo (2008), una educación de calidad es aquella que logra que los estudiantes aprendan lo que deben aprender en el tiempo estimado, y de la mejor manera, haciendo un uso racional de los recursos de que dispone el sistema educativo, es decir, un centro educativo de calidad es aquel que hace que sus estudiantes aprendan de la mejor manera, aprovechando todos los recursos disponibles en la institución.

Para definir la calidad de la educación deben tomarse en cuenta una serie de indicadores.

Garbanzo (2008) considera que los principales indicadores, con miras a la búsqueda permanente de elevar los niveles de calidad en la educación, y que interesan para el presente estudio, son:

- El nivel académico y la disponibilidad del profesorado.

- El acceso equitativo de la población estudiantil.

- Los sistemas de beneficios estudiantiles para las poblaciones menos favorecidas socioeconómicamente.

- La caracterización de la población estudiantil. 
- Los procesos administrativos flexibles y dinámicos.

- La articulación con la investigación de alto nivel y su pertinencia con la realidad social.

- Una visión humanista en la formación profesional.

La calidad de la educación es un factor importante e influyente en una organización educativa, aunque no por eso se encuentra exenta de conflictos.

Según Valle (2007) los conflictos de todo tipo han estado presentes siempre en las organizaciones humanas. El conflicto, como algo inherente a las relaciones interpersonales, es inevitable, por ser las escuelas organizaciones sociales donde perviven y coexisten grupos humanos con propósitos comunes, caracteres y gustos diferentes, es que pasan a ser un espacio de conflictos.

Algunas conductas que originan los conflictos violentos en los colegios son:

- La sociedad es violenta, la violencia ha llegado a adquirir estatus de aceptación y quizá respetabilidad. La historia ha configurado una cultura de violencia.

- La familia es autoritaria y en muchos casos pobre. La pobreza es una forma de violencia.

- La pobreza humilla y la humillación es semilla de violencia.

- La escuela es autoritaria y esto es una manera de ejercer violencia, como por ejemplo a través de castigos físicos o degradantes.

- $\quad$ La TV y el cine con espectáculos violentos.

- Los deportes de alta adrenalina y de confrontación física donde el héroe es la fuerza física

- Los medios transmitiendo y exaltando la violencia.

- Los juguetes de violencia y guerra.

- No se crea tiempo para escuchar a los adolescentes.

- Los adultos dan malos ejemplos.

- La comida chatarra como fuente de agresividad y violencia.

- Precaria cohesión social o coexistencia armoniosa entre clases (Valle, 2007) 
Ante el problema de los conflictos y la violencia lo que más preocupa es si el personal docente y administrativo se encuentran preparados para enfrentar el problema de la forma más acertada y si están abordando el conflicto como lo estipula el debido proceso, sin obviar el objetivo formativo de su abordaje.

\subsubsection{Conflictos estudiantiles su conceptualización}

La violencia se percibe como un problema mundial que se ha integrado en todos los sectores de la sociedad, inclusive el educativo.

El conflicto, por tanto, se refiere a la existencia de diferentes perspectivas de la realidad sin que precisamente exista en el manejo de tales diferencias la violencia, es decir, un conflicto mal abordado puede desencadenar en violencia, pero no siempre un conflicto implica violencia.

En cuanto a la influencia del medio en las conductas conflictivas estudiantiles es evidente, aunque no determinante, que el entorno socioeconómico de los estudiantes y sus familias (alto nivel de paro, barrios o poblaciones con infraestructura más deficientes, familias desestructuradas y despreocupadas, entre otras) tiene un peso significativo en el nivel de conflictividad donde el profesorado no tiene capacidad de influencia y cuya intervención debe venir desde otros profesionales. Por lo tanto, una gran parte de los conflictos puede tener origen en el propio alumno y sus familias, pero otros surgen a causa del funcionamiento de las organizaciones educativas, centros de la carencia de estrategias de prevención y atención a la diversidad, según lo mencionan Casamayor, Antúnez, Almejach, Checa, Giné, Guitart, Notó, Rodón, Uranga y Viñar (1999).

Es importante atender a tiempo aquellas situaciones que pueden desencadenar en conflicto, contextualizándolo y encausándolo, de manera positiva, para que le permita al estudiante adquirir aprendizajes para enfrentar situaciones similares en diversos espacios sociales en que se presenten. Para ello, es necesario proponer acciones concretas de atención en todos los niveles de prevención, mediante alternativas de respuesta a cada situación particular. 


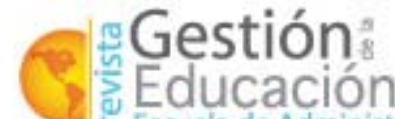 \\ Escuela de Administración Educativa}

Revista Cientifica Digital ISSN:2215-2288

\subsubsection{Alternativas de respuesta a las situaciones conflictivas en los centros educativos}

Dentro de la organización se debe analizar qué comportamientos negativos son los que se producen habitualmente, en qué momento del día, qué alumnos son los que se comportan así habitualmente, número de alumnos, perfil del alumno, a partir de qué edades o niveles comienzan a comportarse de esa forma, cómo reaccionan los compañeros que no adoptan la misma actitud y si se produce contagio, los lugares o espacios comunes donde se presentan mayoritariamente los conflictos; estos cuestionamientos pueden ayudar a definir las estrategias o acciones a seguir, teniendo claros los objetivos aplicados de manera formal para toda la comunidad educativa. (Casamayor, citado por Antúnez, 2000)

Una estrategia a implementar está relacionada con involucrar a toda la comunidad educativa dentro del proyecto que la institución defina para atender la conflictividad en el estudiantado. En relación con ello Casamayor, Antúnez, Almejach, Checa, Giné, Guitart, Notó, Rodón, Uranga y Viñar (1999) recomiendan "bajar los niveles de conflictividad creando canales de comunicación entre padres y alumnos, con la comisión que con ese fin se formó en el centro educativo; recomiendan también mejorar la comunicación con el exterior implementando actividades y jornadas de puertas abiertas” (p.25).

Boqué (citado en Antúnez, Boqué, Casamayor, Cela, Diez de Ulzurrúm, Fajardo, Funes, Garrell, Mainer, Martí, Massaguer, Moreo, Notó, Perales, Rigoll y Tudela, 2000), propone puntos importantes para que esta comisión de convivencia, encargada del proyecto del centro en atención a los conflictos estudiantiles, contemple dentro de sus planes de acción: fomentar actitudes de cooperación, valores y normas que evadan la violencia y busquen la reconciliación, fortaleciendo aspectos positivos mediante la educación en valores.

Por otra parte, sin ser un objetivo, la disciplina puede ser un bien para la convivencia en la escuela y parte del plan que se sugiere. Las normas que están dispuestas en la institución son responsabilidad de las personas encargadas de educar, sin ser una imposición, ni la ostentación del poder, sino tratando que el reglamento sea mayoritariamente compartido, este debe sustentarse en unos principios que la rijan y no en castigos. 
Este trabajo preventivo de los conflictos debe contemplar la sensibilización del personal docente, para que responda oportunamente a los conflictos que se producen en el aula, incorporando dentro del currículo explícito todos los elementos necesarios para que el estudiantado interiorice estas normas como reafirmación de sus derechos. Para ello es importante tener claridad en aspectos como la tipología de los conflictos, de manera que el profesional en educación identifique sus manifestaciones y pueda actuar oportunamente.

\subsubsection{Tipos de conflictos}

Los conflictos se pueden dar en todas las relaciones que las personas intercambian en los diferentes ambientes en que se desenvuelven. En el ámbito educativo las manifestaciones y razones por las que se presenta el conflicto varían de acuerdo con el tipo de relación que mantienen las personas.

Las actitudes y acciones de la administración de los centros educativos determinan en gran parte el éxito del proceso que realice para la resolución del conflicto. Es importante realizar acciones preventivas y de capacitación para no limitar el trabajo al momento en que se presenta el conflicto, dado que los resultados no son igualmente satisfactorios, y para ello se requiere de un trabajo conjunto donde intervenga la comunidad educativa.

\subsection{Sistematización de los resultados y análisis}

Los principales resultados se sistematizan según cada categoría de análisis, de acuerdo con cada uno de los objetivos planteados; posteriormente, se fundamenta teóricamente el análisis de los mismos.

Con respecto a las entrevistas realizadas a los sujetos que participan de esta investigación (administrador de la educación, orientadores, docentes guías, estudiantado), se encontró:

\section{Cuadro $N^{\circ} 1$}




\begin{tabular}{|c|c|}
\hline $\begin{array}{c}\text { Categorías de } \\
\text { Análisis }\end{array}$ & Opiniones de los Sujetos Participantes \\
\hline $\begin{array}{l}\text { 1. Manifestaciones } \\
\text { de los conflictos } \\
\text { estudiantiles que se } \\
\text { generan en el } \\
\text { Colegio Técnico } \\
\text { Profesional de } \\
\text { Jacó. }\end{array}$ & $\begin{array}{l}\text { El administrador de la educación, la orientación, los docentes y el } \\
\text { estudiantado coinciden, en su mayoría, en que las manifestaciones del } \\
\text { conflicto entre estudiantes se da a través de “enjaches”, palabras ofensivas y } \\
\text { obscenas, empujones, grafitis, faltas de respeto, intolerancia, envidia, } \\
\text { agresión física. A criterio de una profesora, existen conflictos que se } \\
\text { manifiestan mediante la burla por discriminación étnica. } \\
\text { Así mismo, estudiantado, docentes y administrador de la educación } \\
\text { coinciden en que las manifestaciones del conflicto entre estudiantes y } \\
\text { docentes y viceversa se muestran en las siguientes acciones: faltas de respeto } \\
\text { (dejar al estudiante hablando solo, no responder el saludo, críticas no } \\
\text { constructivas, tanto hacia el estudiante como hacia otros docentes), uso de } \\
\text { vocabulario irrespetuoso, indirectas para minimizar al estudiante, choteo, } \\
\text { insultos, se alza la voz. }\end{array}$ \\
\hline $\begin{array}{l}\text { 2. Factores internos } \\
\text { que influyen en los } \\
\text { conflictos } \\
\text { estudiantiles. }\end{array}$ & $\begin{array}{l}\text { En concordancia, el estudiantado, docentes y orientadoras mencionan que se } \\
\text { da la desmotivación y descontento en la población estudiantil, debido al } \\
\text { escaso desarrollo académico de las clases, según cita una estudiante "es que } \\
\text { generalmente las críticas que se hacen en tiempo de clase no son } \\
\text { constructivas, yo como profesor no puedo venir a tomar tiempo de las } \\
\text { lecciones de un grupo para desahogarme de lo que le pasó en otro grupo, a } \\
\text { veces duran cuarenta minutos en puro bla, bla, bla, y se les va el tiempo y } \\
\text { terminó la lección y ya se pueden ir, y uno queda como si nada, porque yo } \\
\text { no sabía ni siquiera que el profesor tenía problemas, que lo habían visto en } \\
\text { la calle, entonces uno les dice que es esto, que se está perdiendo el tiempo", } \\
\text { afirman que se da ausentismo de los docentes del aula y de la institución, se } \\
\text { abordan temas que no tienen relación con la materia y que son de carácter } \\
\text { deshonestos, según la percepción de los estudiantes. Además, existe } \\
\text { disconformidad con la metodología utilizada, dado que no se explica la } \\
\text { materia, esto les provoca alejamiento de la realidad escolar y disminución de } \\
\text { la motivación para asistir a las clases. } \\
\text { El personal docente opina que los estudiantes conflictivos manifiestan } \\
\text { desmotivación, irritabilidad, conductas de tipo grotesco, explosivos, } \\
\text { impulsividad, inseguridad, los caracterizan como personas sensibles, capaces } \\
\text { de percibir la inestabilidad de otros. } \\
\text { El director del colegio reconoce que los estudiantes muestran estas } \\
\text { conductas, sin embargo, las ubica como aspectos normales de la } \\
\text { adolescencia. }\end{array}$ \\
\hline & todos los sujetos participantes en \\
\hline
\end{tabular}




\begin{tabular}{|c|c|}
\hline $\begin{array}{c}\text { Categorías de } \\
\text { Análisis }\end{array}$ & Opiniones de los Sujetos Participantes \\
\hline $\begin{array}{l}\text { 3. Estrategias que } \\
\text { utilizan los } \\
\text { docentes, } \\
\text { orientador y } \\
\text { administrador } \\
\text { educativo en la } \\
\text { resolución de los } \\
\text { conflictos } \\
\text { estudiantiles. }\end{array}$ & $\begin{array}{l}\text { cuanto a que no existen medidas de prevención del conflicto. } \\
\text { Sobre el abordaje y seguimiento, el administrador de la educación menciona } \\
\text { que la dirección no tiene un papel directo, más bien, son las orientadoras } \\
\text { quienes se encargan de mediar para apaciguar a los involucrados y de } \\
\text { comunicar al padre, madre de familia o encargado de los estudiantes } \\
\text { implicados, tratan de escucharlos, ayudarlos y de aplicar el Reglamento de } \\
\text { Evaluación de los Aprendizajes del Ministerio de Educación Pública. } \\
\text { En cuanto a programas de capacitación, dirigidos al personal docente y } \\
\text { estudiantes es consistente, desde la opinión de la administración y del } \\
\text { estudiantado, que no existen tales programas; sin embargo, algunos docentes } \\
\text { y orientación señalan que sí se dan talleres y charlas sobre el tema del } \\
\text { abordaje del conflicto. } \\
\text { Sobre los canales de comunicación propuestos por la institución para } \\
\text { mantener relación con padres, madres de familia o encargados, el director } \\
\text { menciona que sí existe anuencia para recibirlos, pero no hay un plan } \\
\text { estructurado que fomente o impulse esta medida. } \\
\text { Acerca de la existencia de un manual que establezca normas de convivencia } \\
\text { en el centro, los profesores y el administrador educativo manifiestan que no } \\
\text { lo conocen, otros docentes mencionan que si existe el reglamento interno, } \\
\text { pero que no se aplica, y que en su elaboración no hay participación de los } \\
\text { estudiantes. }\end{array}$ \\
\hline $\begin{array}{l}\text { 4. Problemas } \\
\text { sociales de la } \\
\text { comunidad de } \\
\text { Garabito. }\end{array}$ & $\begin{array}{l}\text { Existe un acuerdo entre estudiantes, docentes, orientación y administrador de } \\
\text { la educación, al mencionar que los principales problemas sociales que } \\
\text { enfrenta la comunidad de donde proviene el estudiantado son drogadicción } \\
\text { (consumo y venta), desintegración familiar, explotación sexual comercial, } \\
\text { prostitución, diferencia de clases sociales, pobreza, influencia de cultura } \\
\text { extranjera, homosexualismo. }\end{array}$ \\
\hline
\end{tabular}

Es importante mencionar que en la manifestación de los conflictos estudiantiles intervienen causas macro sociales. Sin embargo, este estudio se limitó a indagar las causas internas en la organización, debido a que son las que están afectando, y que de alguna forma son generadas por las anteriores. Para determinar las principales manifestaciones del conflicto estudiantil entre estudiantes-estudiantes y docentes-estudiantes en el Colegio de Jacó, se cotejó la información proveniente del trabajo de campo de esta investigación. 
Los conflictos están relacionados con situaciones cotidianas de la vida social y escolar, según lo expresan Casamayor y Mainer (citado en Antúnez, Boqué, Casamayor, Cela, Diez de Ulzurrúm, Fajardo, Funes, Garrell, Mainer, Martí, Massaguer, Moreo, Notó, Perales, Rigoll y Tudela, 2000). Sin embargo, un conflicto que no es abordado desde sus inicios, de una forma preventiva, puede desatar la violencia, pues así es percibido por los sujetos participantes en esta investigación, al mencionar que al no haber abordaje directo con las personas que tienen conflictos se desencadenan agresiones físicas más o menos violentas.

Al consultárseles sobre la forma de intervención que hacen los funcionarios en los conflictos estudiantiles, los estudiantes afirman: "nada, nada más llegan ahí como a ver...” "y digamos hacen algún comentario y algo y el profe sale a buscar a Magdalena (orientadora), siempre, él no puede resolver el problema, no puede decir qué pasa, por qué son así, por qué actúan así, no! de una vez van, buscan a Magdalena, ella viene y dice chiquillos que es lo que les pasa, dejen ese comportamiento así, al profesor no le gusta, y es lo único que nos dice, no nos dicen en realidad que es lo que nos molesta o cual es la actitud que el profesor está tomando hacia nosotros, qué nos está haciendo actuar de esa manera. Pero nosotros somos siempre los de la culpa, nosotros somos los malcriados, nunca ven por qué somos así con esos profesores”.

Así mismo, el director manifiesta que entre estudiantes inclusive "se han agredido, se han agarrado del pelo, se han ido al suelo, se dicen toda clase de palabras”

Las afirmaciones anteriormente citadas, evidencian que la administración de la educación en la organización educativa del estudio no llena las expectativas de los estudiantes, y evidentemente no se está atendiendo el conflicto, se trata de tranquilizar a los involucrados en el momento, pero no se aborda desde la raíz, se deja claro que existe, para los estudiantes, un vacío en cuanto a la atención de las situaciones que experimentan dentro de la organización educativa con los diferentes actores, incluyendo al personal docente y administrativo.

Las principales manifestaciones del conflicto en la organización educativa en estudio son: "enjaches, uso de palabras ofensivas y obscenas, grafitis, faltas de respeto que se dan de los profesores hacia los estudiantes, de los estudiantes hacia los docentes y entre los estudiantes a través del choteo, insultos, gritos, vocabulario irrespetuoso, dejar al 
estudiante hablando solo o no responder un saludo, también se da por parte del docente las críticas no constructivas (así percibidas por el estudiantado) hacia estudiantes y hacia otros docentes”, todas estas manifestaciones de conductas conflictivas en forma reiterada podrían iniciar actos violentos.

Las confrontaciones, peleas, uso de palabras negativas, el maltrato verbal por medio de los insultos, hablar mal de alguien o ignorarlo. Indudablemente estas manifestaciones impiden alcanzar la máxima calidad de los servicios administrativos y pedagógicos que se brindan en las organizaciones educativas con el propósito de lograr que la población estudiantil adquiera habilidades y valores que respondan a lo planteado en la Constitución Política y en los fines de la educación costarricense.

Al respecto, Chiavenato (2006) señala que la administración de la educación debe integrar los recursos humanos y materiales y dirigirlos en función del alcance de los objetivos organizacionales.

El profesional en administración de la educación debe tomar conciencia de la problemática presente en su organización, así como de los sujetos involucrados en el conflicto y actuar de forma estratégica, como lo afirman García, Rojas y Campos (2002) al expresar que una de las estrategias en la administración, de la educación que se puede implementar para el abordaje efectivo de las situaciones conflictivas dentro de las organizaciones educativas, puede estar relacionada con la toma de decisiones.

En el caso del Colegio Técnico Profesional de Jacó la mayoría se da por discriminación étnica, según se evidencia, ya que los encuestados así lo perciben al expresar que existen "Rencillas juveniles, algunas veces por celos entre ellos, conflictos por no aceptar específicamente a los nicaragüenses, o sea, rechazo a los extranjeros”

Posterior al trabajo de campo aplicado en el Colegio Técnico Profesional de Jacó y la aplicación de los instrumentos utilizados durante la investigación, se evidencia la presencia de algunos factores internos que influyen en los conflictos estudiantiles, tales como desmotivación, descontento, explosividad, impulsividad, agresividad, enojo, entre otros. Al cuestionar a los estudiantes sobre conductas y actitudes de las personas que se involucran en conflictos, ellos manifiestan “...eso de ser explosivos y agresivos son problemas que muchas veces son problemas que uno trae de la casa y ellos creen que 
mandando boletas y expulsando van a solucionar el problema, en lugar de sentarse y preguntarle a uno ¿qué le pasa, en qué le puedo ayudar?”.

Lo anterior, confirma la teoría expuesta por Massaguer (citado por Antúnez, Boqué, Casamayor, Cela, Diez de Ulzurrúm, Fajardo, Funes, Garrell, Mainer, Martí, Massaguer, Moreo, Notó, Perales, Rigoll y Tudela, 2000), al mencionar que el conflicto estudiantil se debe, en algunos casos, a lo inadecuado del proyecto educativo, a las características de la etapa, o también por una inconveniente metodología generadora de tensiones, con la cual son desarrolladas las clases, tal y como lo manifiestan los estudiantes entrevistados, sobre la inconformidad que sienten con respecto al ausentismo del docente al aula y a la institución, así como de las prácticas pedagógicas catalogadas por ellos como desmotivantes, por ser magistrales y memorísticas.

Por tanto, los conflictos estudiantiles se deben no solo a las situaciones del entorno o de la relación cotidiana entre las personas, sino también, a los factores o características propias de la adolescencia, esto se puede deducir de las opiniones de los sujetos entrevistados. Es importante para la administración de las organizaciones educativas de secundaria se tome en cuenta la etapa de desarrollo y el contexto social en que se encuentra la población estudiantil de su institución, es decir, considerar que el surgimiento de los conflictos se puede generar desde la influencia del contexto sociocultural o por condiciones o situaciones propias de la etapa de la adolescencia, pues esto le permitirá comprender mejor a esa población y, por ende, abordar el conflicto que surge, tanto entre estudiantes como entre docentes y estudiantes.

Con respecto, a las estrategias para la resolución de conflictos desde la administración de la educación, es importante que el personal directivo posea características, habilidades y capacidades que contribuyan al cumplimiento de funciones que le permitan llevar a cabo, de manera exitosa, su accionar dentro de la institución. Dentro de las principales funciones, Chiavenato (2006) destaca los procesos de planificación, organización, dirección y control en el uso de los recursos para lograr los objetivos organizacionales.

Al respecto Casamayor (1999) sugiere que para la atención del conflicto, es necesario estructurar un plan de atención desde la prevención, abordaje y seguimiento; para ello propone, entre algunas posibilidades, formar una comisión que promueva la sana 


\section{Gegestión}

Revista Cientifica Digital ISSN-2215-2288

convivencia, diagnosticando los comportamientos negativos que se producen en el centro en relación con el conflicto. Se sugiere que la comisión promueva canales de comunicación entre los padres de familia, población estudiantil y el personal de la organización educativa, para ello recomienda actividades como jornadas de puertas abiertas, celebraciones de actividades abiertas al público. Así mismo, recomienda la formación de grupos especializados en técnicas de mediación y negociación, constituidos por estudiantes y docentes, utilizando diferentes técnicas que proporcionen herramientas para prevención y resolución de los conflictos que puedan presentarse.

Sin embargo, en el Colegio Técnico Profesional de Jacó, según expresan los sujetos participantes, no existe un plan de prevención del conflicto; no se da el abordaje y seguimiento del mismo por parte de la administración de la educación, de los docentes, ni de la orientación. En algunos casos se aplica el Reglamento de Evaluación de los Aprendizajes, el cual no propone estrategias de prevención, abordaje o seguimiento del conflicto, sino que más bien, según la opinión del estudiantado participante, es sancionador. Al respecto, Casamayor (citado por Antúnez, 2000) señala que la propuesta del manual de convivencia debe integrar algo más que las prohibiciones, dando relevancia a lo que se espera de la población estudiantil en la organización educativa.

Antúnez, Boqué, Casamayor, Cela, Diez de Ulzurrúm, Fajardo, Funes, Garrell,

Mainer, Martí, Massaguer, Moreo, Notó, Perales, Rigoll y Tudela (2000), recomiendan instruir a la comunidad educativa en temáticas que fomenten la sana convivencia. Sin embargo, en la organización estudiada solo hay capacitación dirigida a los orientadores en su formación profesional, no así para a los docentes ni para la comunidad estudiantil, observándose en el currículo la ausencia de capacitación en materia del manejo de conflictos.

En el Colegio Técnico Profesional de Jacó, según mencionan las personas participantes de la investigación, el manual de convivencia no existe, en su lugar se encuentra la normativa interna, que es un documento que contiene la definición de normas propias de la institución.

Por lo tanto, según los resultados obtenidos en la investigación realizada, es evidente la falta de equidad en la distribución del poder, donde las decisiones y el control 
son ejercidos por la parte administrativa y docente, sin tomar en cuenta la opinión de la comunidad estudiantil.

La organización en estudio se encuentra inmersa en un contexto social geográfico influenciado por el turismo costero y con problemas sociales como: la drogadicción (consumo y venta), desintegración familiar, explotación sexual comercial, prostitución, diferencia de clases sociales, pobreza, influencia de cultura extranjera, homosexualismo, los cuales repercuten en el proceso de enseñanza aprendizaje del estudiantado, generando una serie de conflictos, evidenciado en el diagnóstico institucional y las manifestaciones en las entrevistas realizadas a los diferentes sujetos.

Acerca de la identificación de estos problemas sociales en la comunidad de Garabito, uno de los docentes encuestados menciona: “Incorporación de costumbres extranjeras, prostitución, consumo de drogas”, al respecto una de las orientadoras también señala “drogadicción, proxenetismo, problemas familiares, prostitución, aculturación, pobreza, homosexualismo y lesbianismo”. Es el motivo por el cual se expresa que el contexto influye en el interactuar social del alumnado.

Por lo tanto, no puede haber educación de calidad, si no se toman en cuenta características propias del contexto geográfico, cultural y social, pues las mismas determinan la peculiaridad del estudiantado.

Según Garbanzo (2008) para elevar los niveles de calidad es importante tomar en cuenta el nivel académico y la disponibilidad del profesorado, así como también la caracterización de la población estudiantil. Es evidente que la organización en estudio no cuenta con un instrumento que tome en cuenta las particularidades, tanto sociales como geográficas, de la población estudiantil ni del contexto social y cultural en que se desenvuelve.

No existe ningún plan que atienda las necesidades del Colegio Técnico Profesional de Jacó, donde se minimicen las debilidades y amenazas, apoyándose en las fortalezas y oportunidades. Esta es más de las ocasiones más importantes y urgentes que se debe a retomar desde la administración de la educación, razón por la cual se plantea una propuesta que tome en cuenta las particularidades del contexto para prevenir de forma oportuna los conflictos estudiantiles. 
Una vez, analizado los resultados de la investigación, es posible ofrecer un modelo de prevención de conflictos estudiantiles para este tipo de organizaciones.

\subsection{Modelo Estratégico de prevención de conflictos estudiantiles}

Se presenta, a continuación, un esquema que representa los pasos necesarios para la atención preventiva de los conflictos estudiantiles, dentro de los cuales se deberá realizar una serie de etapas de concienciación, sensibilización, capacitación, seguimiento y evaluación dentro del programa de prevención de los conflictos estudiantiles, donde se involucra a toda la comunidad educativa: administración de la educación, departamento de orientación, personal docente, estudiantes, padres y madres de familia. Se considera vital, la participación de especialistas de instituciones que de una u otra forma son implicadas en la temática. 


\section{- Gestión \\ Modelo Estratégico de Prevención de Conflictos Estudiantiles}

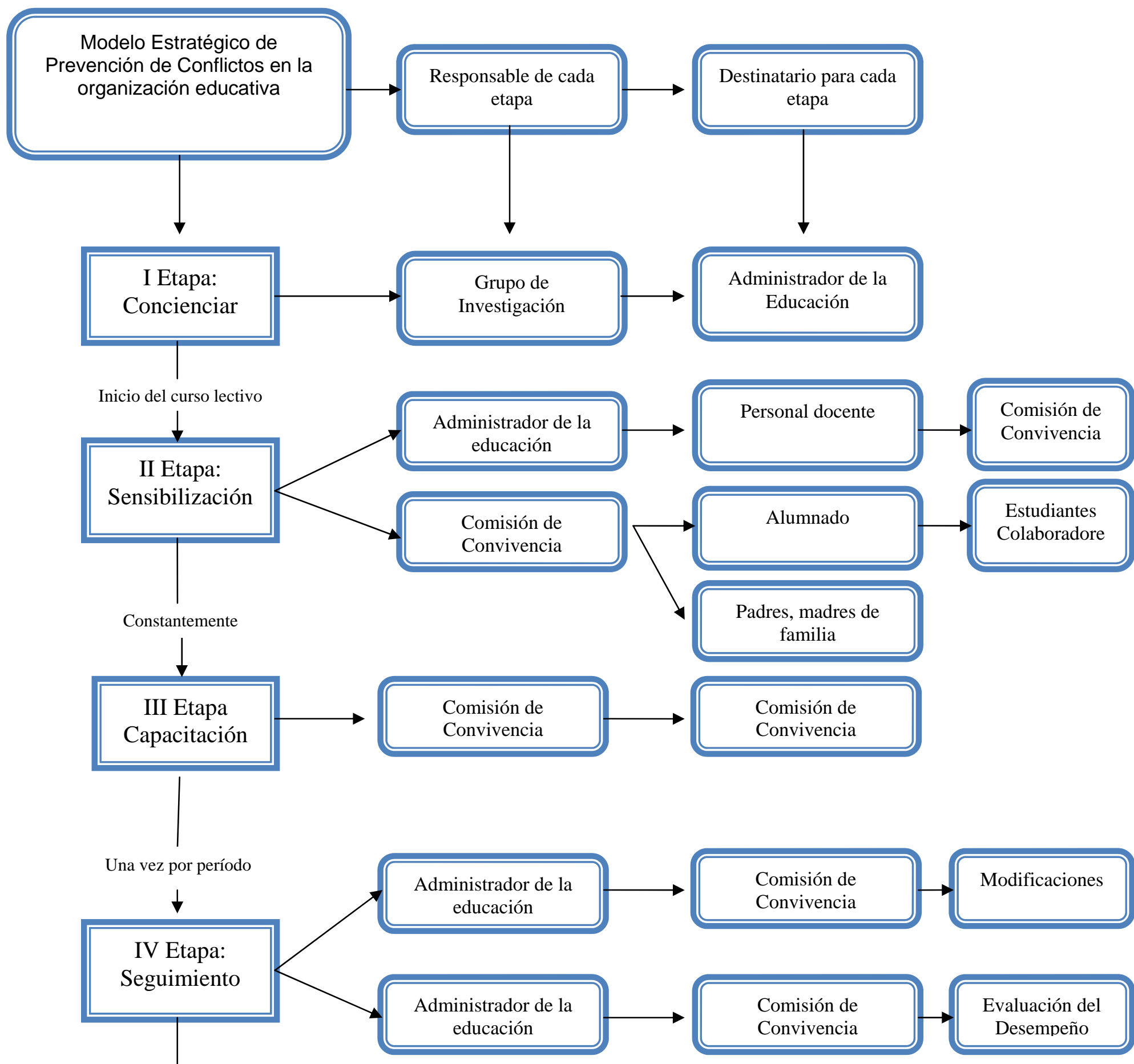

Final de curso lectivo

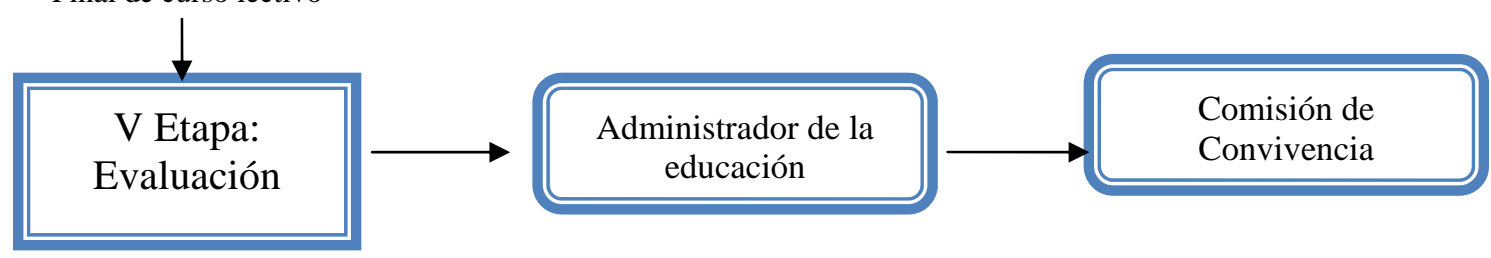




\subsection{Conclusiones}

- Es evidente que la gestión administrativa del Colegio Técnico Profesional de Jacó, durante el año 2008, tuvo importantes carencias para atender preventivamente los conflictos estudiantiles desde las funciones de planificación, organización y seguimiento.

- A pesar de que han sido identificadas las principales manifestaciones de conflictos estudiantiles, no se ve interés o preocupación por parte de la administración de la educación en la creación y aplicación de un plan estratégico que aborde esta y otras necesidades detectadas en el diagnóstico institucional.

- Se evidencia en el trabajo de campo falta de compromiso y conciencia por parte del profesorado y de la administración de la educación en cuanto a la prevención, intervención y resolución del conflicto en forma pacífica, reflejando la desatención hacia el estudiantado, que es hacia donde dirigirse los esfuerzos de formación constante.

- El estudio muestra que no existe promoción del desarrollo profesional de los docentes, ni se le brinda herramientas de capacitación a los estudiantes ni a los docentes para resolver las situaciones conflictivas.

- Las conductas de irrespeto de los docentes hacia el estudiantado influyen en la formación de conflictos entre estudiantes-docentes, esto es un aspecto que continúa vigente, a pesar de que esta situación ha sido comunicada ante el administrador de la educación del Colegio Técnico Profesional de Jacó.

- A nivel administrativo hay disponibilidad para atender al estudiantado. Sin embargo, existe una debilidad en la toma de decisiones y en la puesta en marcha de acciones pertinentes.

- El instrumento disciplinario para la corrección de conductas es la aplicación de boletas, cuyo modelo es emanado del Ministerio de Educación Pública, y se evidencia que las mismas no han dado ningún resultado positivo en el abordaje del conflicto; por el contrario, estas empeoran la situación porque no contribuyen a su resolución, sino que 
tienen una misión sancionatoria que provoca actitudes desafiantes por parte de los estudiantes.

- Según lo expresado por los sujetos de la investigación y por los autores consultados, existe relación entre los niveles de conflictividad en el centro educativo y los problemas del contexto social, a lo cual se le debe implementar acciones de tipo administrativas y pedagógicas que contribuyan en la formación integral del estudiantado, para cumplir así con uno de los fines de la calidad de la educación costarricense.

\section{Referencias Bibliográficas}

Antúnez, S., Boqué, M.C., Casamayor, G., Cela, J., Diez de Ulzurrúm, A., FajardoP., Funes, J., Garrell, T., Mainer, J., Martí, J., Massaguer, M., Moreo, A.C., Notó, F., Perales, P., Rigoll, A. y Tudela, P. (2000). Disciplina y Convivencia en la Institución Escolar. Barcelona, España: Editorial GRAO.

Apple, M. (1997). Teoría Critica y Educación. Argentina: Niño y Dávila.

Argudín, Y. (2006). Educación Basada en Competencias. México: Editorial Trillas.

Barrantes, R. (2005). Investigación: un Camino al Conocimiento. Costa Rica: EUNED

Carr, W. (1996). Una Teoría para la Educación. España: Morata.

Casamayor, G., Antúnez, S., Almejach, R., Checa, P., Giné, N., Guitart, R., Notó, C., Rodón, A., Uranga, M. y Viñas, J. (1999). Cómo dar respuesta a los conflictos: La Disciplina en la Enseñanza Secundaria. Barcelona, España: Editorial GRAO.

Chiavenato, I. (2006). Introducción a la teoría general de la administración. México: Editorial McGraw. Hill Interaméricana.

Delval, J. (2002). La escuela posible. España: Pirámide.

Durán, F. (mayo, 2004). Escuela y disciplina. Periódico La Nación, p.34. San José, Costa Rica.

Garbanzo, G. (2008). Calidad y Equidad de la Educación Superior Pública. Aspectos por considerar en su interpretación. Revista Educación, 31(2)11-27. Universidad de Costa Rica. San José, Costa Rica.

García, J. y García, A. (1996). Teoría de la Educación. España: Salamanca. 
García, N., Rojas, M. y Campos, N. (2002). La Administración escolar: para el cambio y el mejoramiento de las instituciones educativas. San José, Costa Rica: Editorial Universidad de Costa Rica.

Martínez, C., Orozco, V. y Vargas, A. (2000). Liderazgo, Relaciones de Poder y Resolución de Conflictos en Instituciones Educativas. San José, Costa Rica: MEP, UCR.

Rodríguez, E. (2001). Cinco Educadores en la Historia. San José, Costa Rica: EUNED.

Rojas, Y. y Barahona, M. (2006). Hacia un modelo educativo para elevar la calidad de la educación costarricense: Una propuesta de estrategias, políticas y acciones. San José, Costa Rica: EUNED.

Sanvisens, A. (1984). Introducción a la pedagogía. Barcelona: Barcanova.

Valle, V. (junio, 2007). Conflictos Estudiantiles en las Organizaciones Educativas. Panel en el Auditorio de la Facultad de Educación. Universidad de Costa Rica. San José, Costa Rica. 